\title{
The Different Clinical Presentations of Allergic Fungal Rhinosinusitis
}

\author{
Noorah AL Mulhem ${ }^{1 *}$, Ali Al Momen ${ }^{1}$ and Fadhel Almolani ${ }^{2}$ \\ ${ }_{1}^{1}$ Department of Surgery, King Fahad specialist hospital, Saudi Arabia \\ ${ }^{2}$ Department of Radiology, King Fahad Specialist Hospital, Saudi Arabia
}

*Corresponding author: Noorah AL-Mulhem, Resident trainee, ORL-HNS section, Department of Surgery, King Fahad specialist hospital- Dammam 31444, Saudi Arabia, Tel: 00966506889889; Email: noura.j.almulhem@gmail.com

\section{Abstract}

The aim of this study is to describe different clinical presentations of allergic fungal rhinosinusitis (AFRS) managed in King Fahad Specialist hospital, a tertiary care hospital in the Eastern Province, Saudi Arabia. A total number of 15 cases 8 males and 7 females) of AFRS were diagnosed and managed. The youngest age at presentation was 7 years and the eldest was 68 years. 7 of these cases were briefly presented in this article, each with a different clinical presentation and all diagnosed to have allergic fungal sinusitis based on Bent and Khun diagnostic criteria. Some of these cases even have been diagnosed with other types of fungal sinusitis that were occurring concurrently. To conclude, despite AFRS being categorized as a benign, non-invasive disease, its presentation can range from simple nasal obstruction to signs and symptoms of intraorbital and/or intracranial complications; with paediatric cases being more aggressive in nature than in adults. Other forms of fungal sinusitis may coexist with allergic fungal sinusitis as well. For that, each patient should undergo careful clinical evaluation, detailed histopathological examination to rule out mixed types and malignancies, and a lifelong follow up to manage the recurrence.

Keywords: Allergic Fungal Rhinosinusitis; Histopathological examination; Malignancies

Abbreviations: AFRS: Allergic Fungal Rhinosinusitis; ESS: Endoscopic Sinus Surgery; CSRsNP: Chronic Rhinosinusitis without Nasal Polyps; CRS: Chronic Rhinosinusitis; KFSHD: King Fahad Specialist Hospital.

\section{Introduction}

Fungal sinusitis is classified as aggressive and nonaggressive. These categories were developed in the last decade. The importance of this classification resides in recognizing the type of the fungal sinusitis in terms of the severity in order to plan for the appropriate surgery [1,2]. Allergic fungal rhinosinusitis (AFRS), is a noninvasive fungal disease of the sinuses that was described within the past 40 years. Currently, it is considered as a distinct form of chronic rhinosinusitis (CRS) [3]. It is usually seen in $5 \%-10 \%$ of adults with chronic rhinosinusitis who require surgery [4]. 


\section{Otolaryngology Open Access Journal}

The disease was first recognized in 1976 when Safirstein published the first description of AFRS [5]. He observed the clinical similarity that this assemblage of findings shared with allergic bronchopulmonary Aspergillosis [5,6]. Katzenstein, et al. later on further described 7 similar cases, which was termed as "allergic Aspergillus sinusitis" [7]. It is interesting to find that several reports in the available literature have described AFRS with different clinical presentations; some even involving more than 1 type of fungal rhinosinusitis occurring concurrently $[8,9]$.

In this article, we will review several cases of AFRS, each with different clinical presentation. All were diagnosed and managed in King Fahad Specialist Hospital (KFSHD), a tertiary care hospital in Al-Dammam, Saudi Arabia.

\section{Materials and Methods}

Case series study, the data was collected retrospectively. All cases were diagnosed based on Bent and Khun diagnostic criteria of AFRS. A total of (15) different cases were identified and 7 different cases will be presented in this article. All cases were managed at King Fahad Specialist Hospital in Dammam, Saudi Arabia (KFSH-D) during the period of (2009-2017) after an institutional review board approval was obtained. The clinical presentations, histopathology results, and imaging studies were reviewed. All cases underwent surgical and medical management with close follow up post treatment.

\section{Results}

8 males and 7 females were identified. The youngest age was 7 years and the eldest was 68 years, the mean was 23 years.

These two tables summarize the different clinical presentations of AFRS in both adult and pediatric populations (Figures 1A \& B).

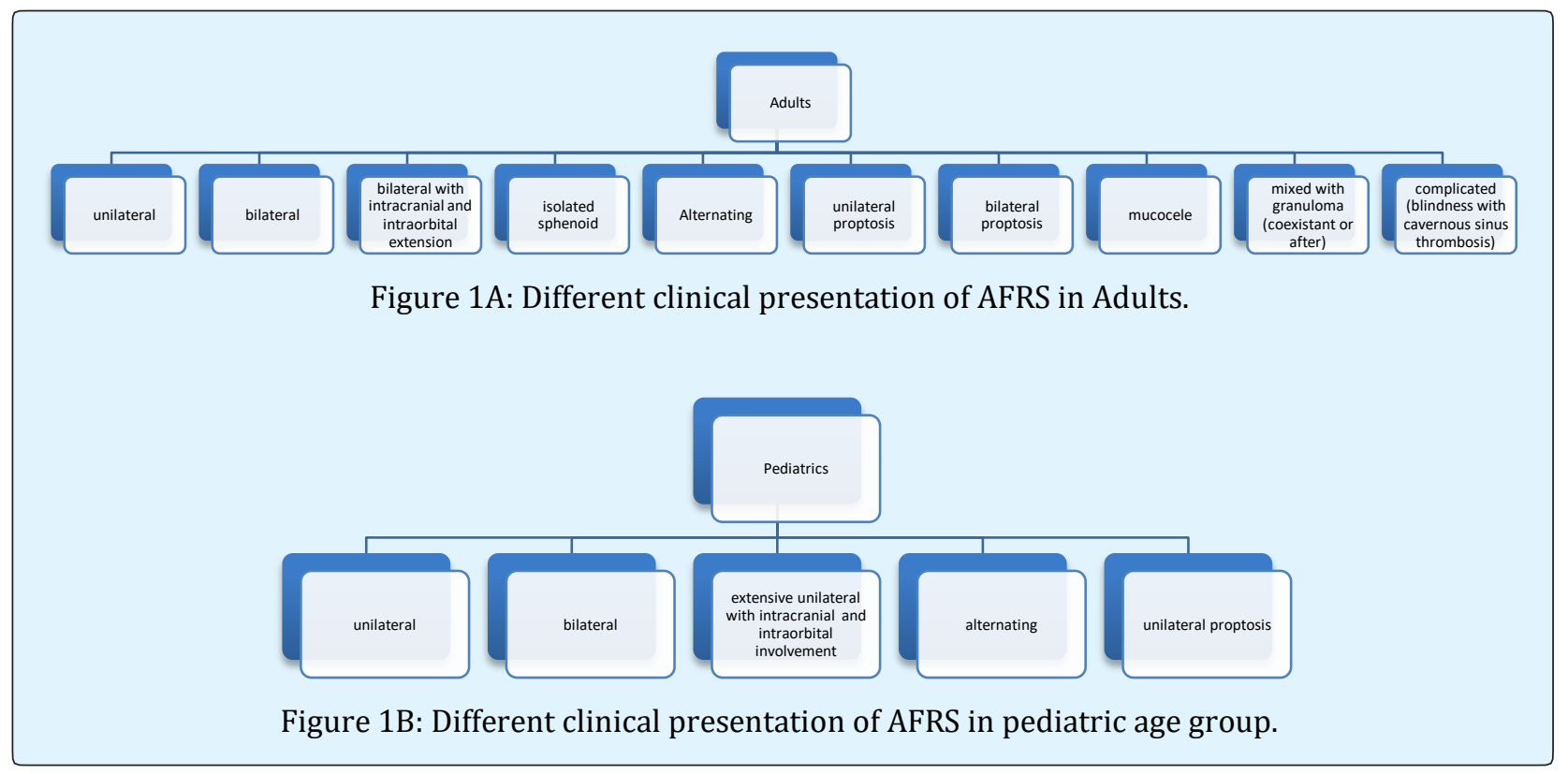

\section{Illustrative Cases}

Case 1: AFRS with Blindness: 23 years old Saudi female who is known to have eczema was referred from another hospital as a case of AFRS with intracranial and intraorbital extension. Patient presented initially with history of nasal discharge that started 3 months prior and was associated with post nasal drip. There was a positive history of headache and facial heaviness of 2 months duration that was aggravated by leaning forward. The patient noticed to have a decreased vision in the right eye that started 2 months back and the visual loss was progressive to complete loss at the time of presentation. On examination, there were bilateral nasal polyps grade 2 with the thick mucin. Examination of the eyes showed an intact extraocular muscle movement. Immediate imaging 


\section{Otolaryngology Open Access Journal}

was obtained, CT scan showed heterodense contents with bone expansion and dehiscence of the right sphenoid sinus wall and the optic canal, resulting in optic nerve compression (Figures 2A \& B).


Figure 2: AFS with blindness.

Figure 2A: Coronal T1 image without contrast shows complete filling of the sphenoid sinuses with hyperintense contents.

Figure 2B: Coronal CT image shows the hyperdense contents with bone expansion and dehiscence of the right sphenoid sinus wall forming the optic canal, resulting in optic nerve compression.

The patient was admitted immediately and Ophthalmology team confirmed the blindness in right eye secondary to optic atrophy. The patient underwent urgent endoscopic sinus surgery (ESS) which showed bilateral sphenoid sinuses impacted with mucin and mud surrounding the right optic nerve which was decompressed and cleaned from fungal debris. The vision improved from light perception preoperatively to 0.3 visual acuity post treatment.

Case 2: AFRS with Granuloma: A 40 years male complaining of nasal obstruction involving the right more than left for 7 years. Associated with hyposmia, nasal itching that worsens with ibuprofen. Nasal examination showed deviated nasal septum to the left, right grade 2 nasal polyps. CT sinuses showed extensive hyper dense and hypo dense disease of the maxillary, ethmoid and frontal sinuses. Erosion of the inferior wall of the left frontal sinus with intra-orbital extension of the disease into intraconal space (Figures $3 \mathrm{~A} \& \mathrm{~B}$ ).

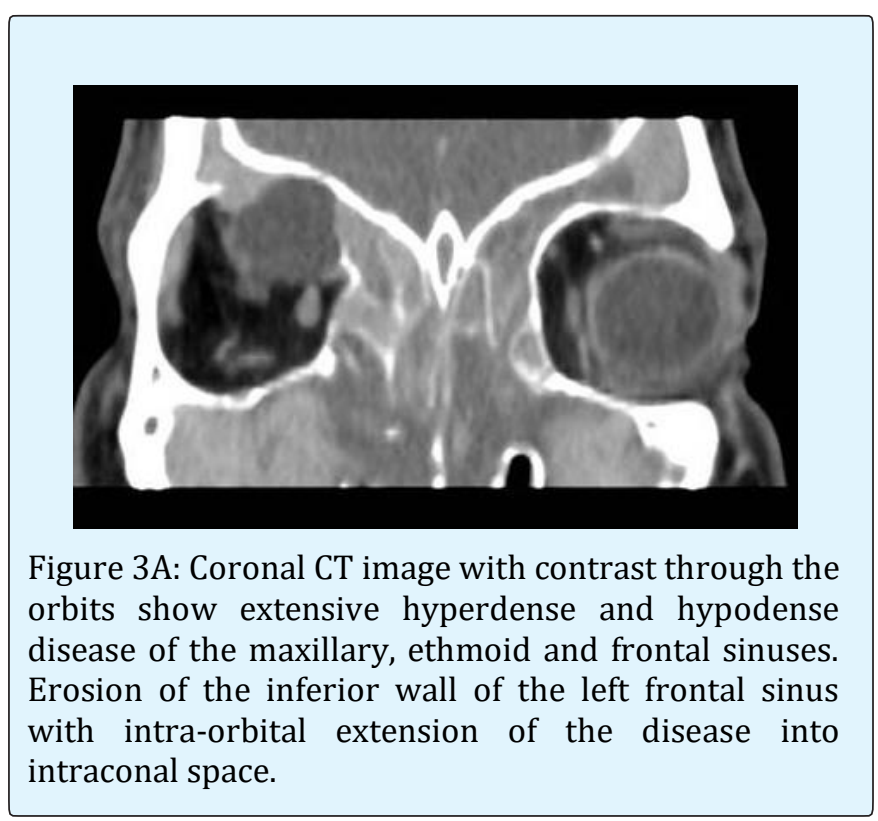




\section{Otolaryngology Open Access Journal}

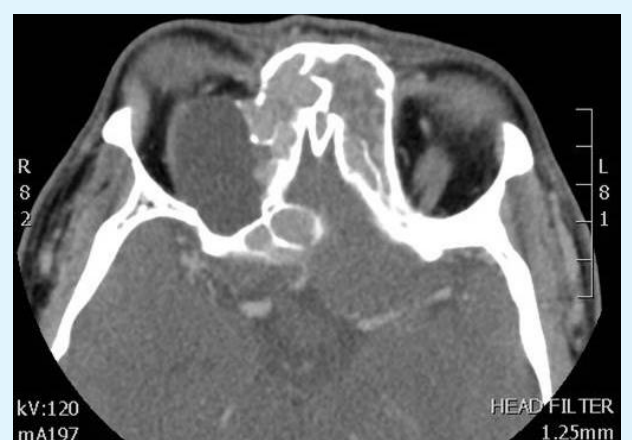

Figure 3B: Axial CT image with contrast through the upper orbits show the right orbital extension of the disease with proptosis as well as the intracranial extension from the posterior ethmoid disease.

Patient underwent Endoscopic sinus surgery, polyps and mucin were removed from sinuses. The left lamina papyrecea was found eroded and cleaned from thick mucin inside the orbit.

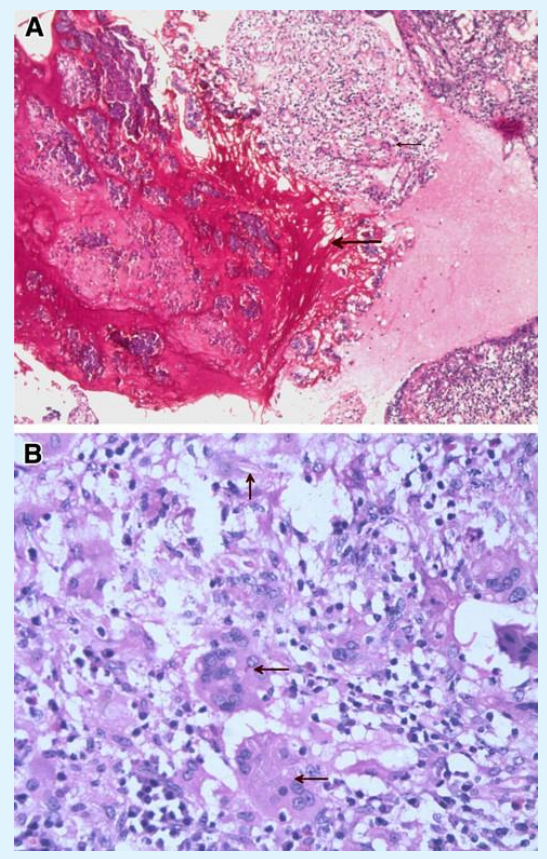

Figure 4A: Photomicrograph demonstrating allergic mucin typical of AFS (big arrow) along with granuloma to us inflammation and giant cells (small arrow) in the adjoining mucosa (H \& E stain).

Figure 4B: High-power view of the same specimen showing foreign body giant cells (arrows) in the mucosa (H \& E stain)
The histopathology report showed inflammatory nasal polyps with multifocal granuloma (Figures 4A \& 4B). Patient showed no recurrence after five years follow up.

Case 3: AFRS with Proptosis: 31 years' old male presented complaining of bilateral nasal obstruction, headache, and post nasal drip for 3 years. The patient started to notice right eye proptosis for the last 2 months. There were no symptoms of allergy, no epistaxis nor a change in vision. Examination showed grade 4 nasal polyps bilaterally with thick mucin Eye examination showed right eye proptosis with intact extraocular muscle movement. CT scan sinuses showed hyperdensity with expansion of right ethmoid air cells and sphenoid sinus. Lateral displacement of right lamina was also noted compressing the right orbital contents causing proptosis (Figure 5).

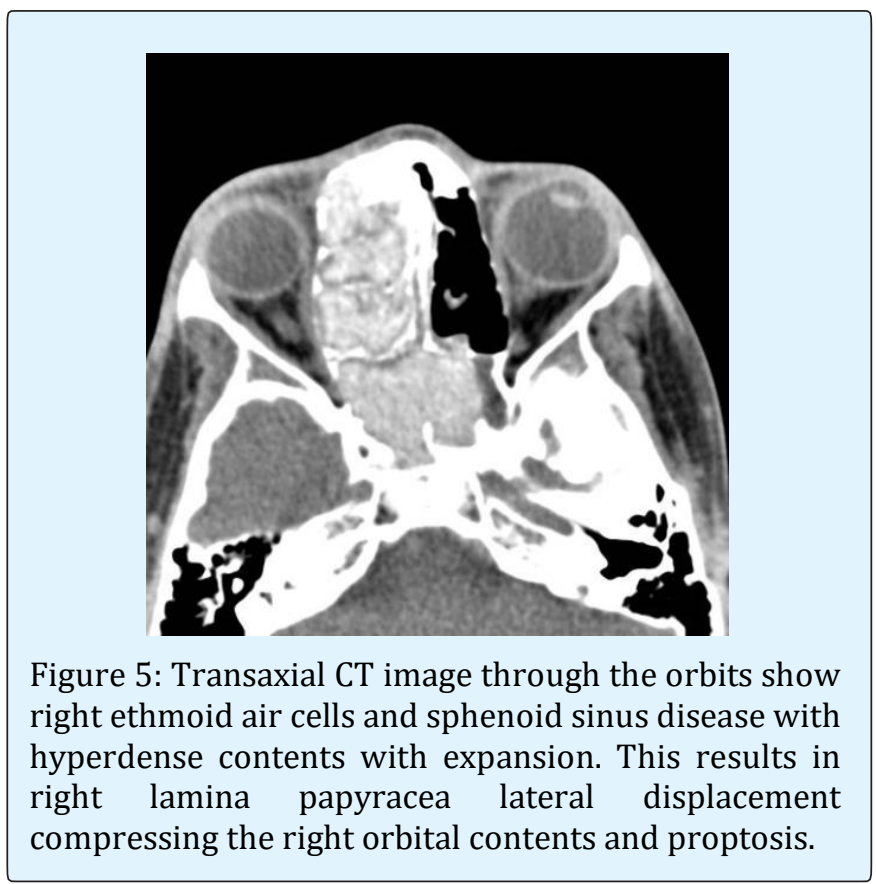

Endoscopic sinus surgery was performed and cleaning of the sinuses from polyps and mucin that causes pressure on the right orbit. The histopathology report showed inflammatory nasal polyps with multifocal granuloma. Patient showed no recurrence after five years follow up.

Case 4: AFRS with skull base extension: A 29-year old asthmatic male complaining of right nasal obstruction that was progressive and associated with rhinorrhea and postnasal drip. Nasal examination showed bilateral nasal polyps. Right eye examination showed right eye proptosis 


\section{Otolaryngology Open Access Journal}

with intact EOM. CT sinuses showed extensive pansinusitis with expansion of the right ethmoid air cells and dehiscence of the cribriform plate and the walls of the sphenoid sinuses resulting in intracranial extension limited by the dura. It extends through both lamina papyracea into the orbit (Figures 6A \& B).

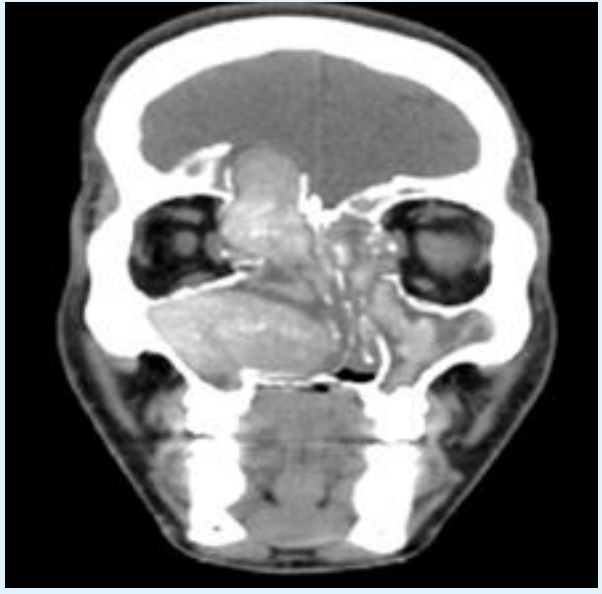

Figure 6A

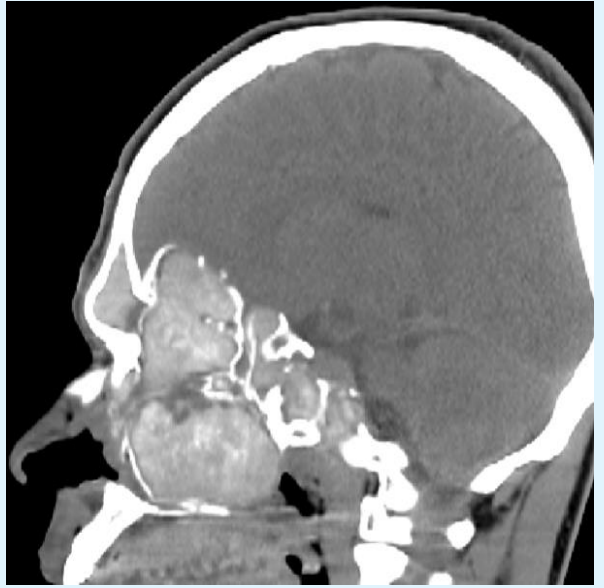

Figure 6B

Figure 6: Coronal (A) and sagittal (B) CT of the paranasal sinuses show extensive pansinusitis with expansion of the right ethmoid air cells and dehiscence of the cribriform plate and the walls of the sphenoid sinuses resulting in intracranial extension limited by the dura. It extends through both lamina papyracea into the orbit. The right maxillary and ethmoid disease expansion obliterates nasal cavity.

Both neurosurgery and ophthalmology teams were consulted. The patient underwent ESS. Endoscopic transnasal sphenoidotomy, bilateral wide antrostomies, cleaning of bilateral frontal sinuses and ethmoids was done. The skull base was devoid of bone with intact dura and no CSF leak observed. The patient showed no recurrence after 2 years follow up.
Case 5: AFRS with Bilateral proptosis: 23 years asthmatic male, morbidly obese, presented with frontal headache, proptosis and epiphora for the last 6 months. Examination showed polyps and mucin. CT scan showed bilateral frontal, ethmoid air cells disease with expansion and bone dehiscence of the roof of the orbits and posterior wall of the frontal sinuses. Intraorbital and intracranial extension and bilateral proptosis is also seen (Figure 7).
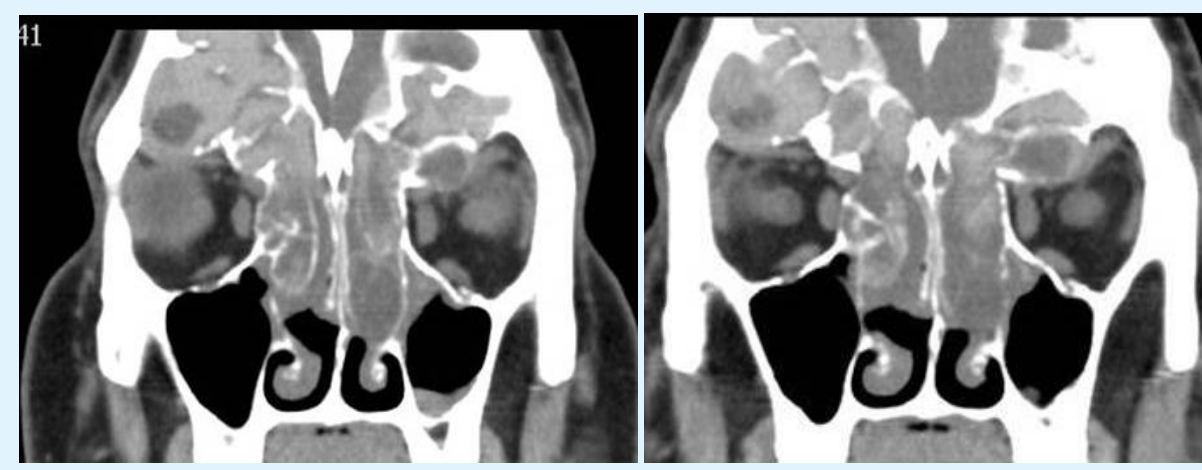

Figure 7: Coronal CT images without contrast show bilateral frontal sinuses and ethmoid air cells disease with expansion and bone dehiscence of the roof of the orbits and posterior wall of the frontal sinuses. It result in intraorbital and intracranial extension and bilateral proptosis. 


\section{Otolaryngology Open Access Journal}

A complete ESS where the sinuses were cleaned and cleared from polyps, mud and mucin. No recurrence was noted after 3 years follow up.

\section{Pediatric Cases}

Case 6: Alternating AFRS: 8 years girl complaining of left nasal obstruction with mild eye proptosis for 9 months noticed by her parents. Examination showed left eye proptosis, enlarged medial canthus. CT sinuses showed left fronto-ethmoidal recess and maxillary disease with bone expansion and obliteration of the left nasal cavity (Figure 8A).

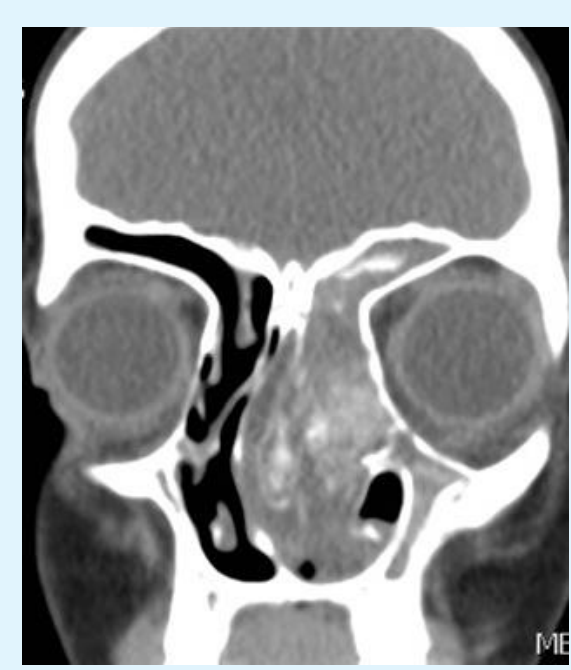

Figure 8A: Coronal CT image show left frontoethmoidal recess and maxillary disease with bone expansion and extension into the nasal cavity associated with nasal polyposis resulting in near obliteration of the nasal cavity and deviation of the nasal septum to the right.

The patient underwent endoscopic sinus surgery and cleaning of the left sinuses from polyps, mud and mucin. Culture was positive for asperigillus. Initially the patient was doing well during her follow ups. However, 1 year later she started to complain of right nasal discharge and obstruction. Examination showed clear left nasal cavity with no recurrence of disease but there were right nasal polyps with mucin. CT sinuses showed recurrence of the disease in the right side with clear left nasal cavity (Figure 8B).

The patient underwent endoscopic sinus surgery for right sinuses cleaning and polyps' removal. Culture was positive for asperigillus. The patient had no recurrence after 3 years follow up.

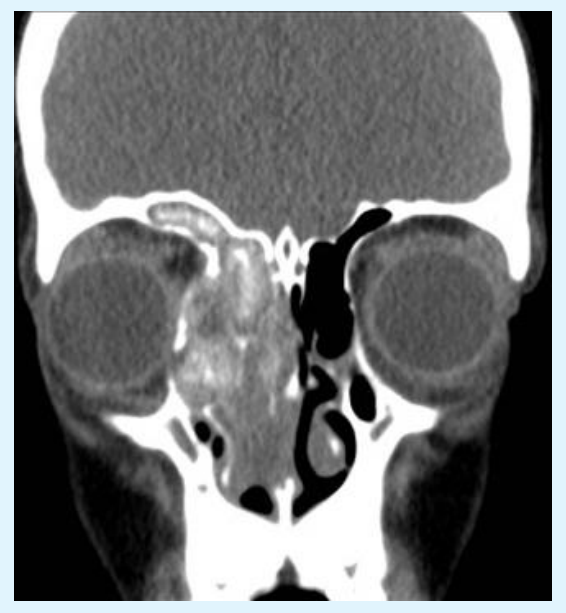

Figure 8B: Coronal CT image post surgery and follow up shows disease recurrence involving the right fronto-ethmoidal recess with expansion and lateral displacement of the right orbit lamina papyracea and nasal polyposis.

Case 7: AFRS with intracranial extradural extension: 15 years old male presented with nasal discharge and obstruction, occasional headache with positive history of allergic symptoms. Examination of the nose showed bilateral nasal polyps.

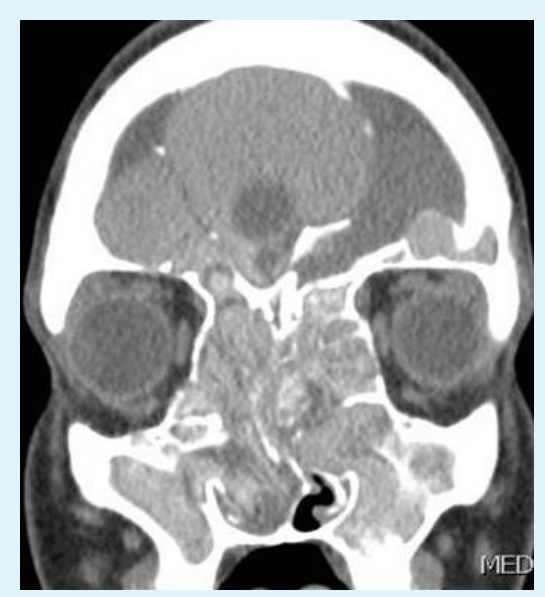

Figure 9A: Coronal CT image through the anterior paranasal sinuses show extensive disease with marked expansion of the sinuses, more pronounced in the right frontal sinus. This results in dehiscence of the posterior wall and intracranial extension. 


\section{Otolaryngology Open Access Journal}

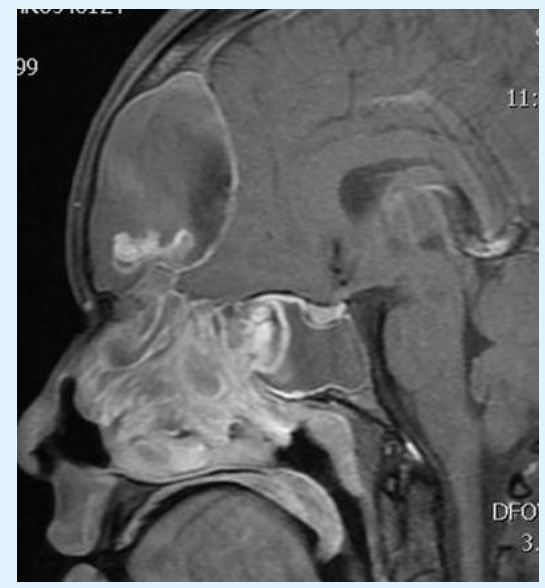

Figure 9B: Sagital T1 post contrast shows the right frontal sinus expansion and intracranial extension limited by an intact dura. The sphenoid sinus expanded with upward displacement of the pituitary fossa.
CT sinuses showed extensive disease with marked expansion of the sinuses with dehiscence of the posterior wall and intracranial extension (Figure 9A). MRI showed right frontal sinus expansion and intracranial extension limited by an intact dura (Figure 9B).

Navigation assisted ESS and cleaning of the sinuses was performed and a dehisecent pulsating dura was cleaned from mucin with no CSF leak observed. The patient noticed to have a recurrent polyps at one year follow up and managed with short course of steroids, no recurrence after 3 years follow up.

\section{Discussion}

Fungal sinusitis has been classified in to invasive and non-invasive form (summarized in Table 1) [3].

\begin{tabular}{|c|c|c|c|c|c|}
\hline \multicolumn{4}{|c|}{ Manifestation of Fungal Rhinosinusitis based on Immunologic status of Host } \\
\hline Host Defense & \multicolumn{2}{|c|}{ Immunodeficient } & Immunocompetent & $\begin{array}{c}\text { Allergic or Altered Cell-Mediated Hyper } \\
\text { responsiveness }\end{array}$ \\
\hline $\begin{array}{c}\text { Manifestation of fungal } \\
\text { rhinusinusitis }\end{array}$ & $\begin{array}{c}\text { Invasive, } \\
\text { fulmitant }\end{array}$ & $\begin{array}{c}\text { Invasive, } \\
\text { chronic }\end{array}$ & $\begin{array}{c}\text { Invasive, } \\
\text { granulomatous }\end{array}$ & $\begin{array}{c}\text { Fungus } \\
\text { ball }\end{array}$ & $\begin{array}{c}\text { Allergic Fungal Rhinosinusitis Nonallergic } \\
\text { eosinophilic fungal rhinosinusitis }\end{array}$ \\
\hline
\end{tabular}

Table 1: Summarises the manifestation of fungal Rhinosinusitis based on the host immunity.

These Invasive forms of fungal disease are usually encountered in immunocompromised subjects. However, some reports have described these forms occurring in immunocompetent individuals [10-12]. Several organizations have classified rhinosinusitis into 4 groups: acute rhinosinusitis, chronic rhinosinusitis with nasal polyps (CSRwNP), chronic rhinosinusitis without nasal polyps (CSRsNP), and allergic fungal rhinosinusitis (AFRS). Thus, categorizing AFRS as distinct form of chronic rhinosinusitis (CRS) [13,14].

Allergic fungal rhinosinusitis (AFRS) has been described within the past 40 years as a noninvasive, benign inflammatory fungal disease of the sinuses [6]. Classically, it develops in young adults and adolescents primarily, being more common in temperate regions with high humidity. Initially, the primary causative organism was thought to be Aspergillus. However, dematiaceous fungi like Alternaria, Bipolaris, Curvularia, and Drechslera have also been implicated $[15,16]$. Allergic rhinitis is found in $63 \%$ of AFRS patients, also $53 \%$ of patients have associated history of asthma [17].
Different clinical presentations of AFRS were described in the literature as seen in our patients. This includes intraorbital and intracranial extension, as well as association with other types of fungal sinusitis. Some cases even presenting clinically with epiphora and eye discharge as a result of nasolacrimal gland obstruction [18]. Generally, though, Patients often complain of symptoms like nasal obstruction, congestion, purulent or clear rhinorrhea, anosmia, and headache $[19,20]$. Many cases present as previously diagnosed CRSwNP that is difficult to treat as well, with history of inhalant atopy. Allergic mucin, that contains sparse numbers of fungal hyphae are seen usually on examination. Investigations show specific IgE antibodies present in serum against Aspergillus species, as well as elevated total serum IgE. As for Imaging; characteristic computed tomography findings include typical heterogeneity of the signal within involved sinuses, complete opacification of at least 1 paranasal sinus, and the expansion and attenuation/erosion of the bone with displacement of adjacent anatomic compartments [21,22]. The bony 


\section{Otolaryngology Open Access Journal}

changes have been assumed to be caused due to expansion of the fungal rather than invasion $[21,23]$ as well as damage from inflammatory mediators $[21,24]$.

However, some sites of mucosal invasion could be missed due to lack of sufficient biopsies from the affected site [25]. Therefore, occult invasion could be the cause of repeated recurrences in patients with AFRS [26]. On MRI, Peripheral enhancement along with a dark center, is characteristic of AFRS. The Reduced central signal intensity on both T1 and T2 images corresponds to fungal concretions as reported by Manning, et al. also, higher signal characteristics at the periphery of involved sinuses corresponds to the inflamed mucosa [27]. Diagnosis of AFRS is achieved using Bent III and Kuhn diagnostic criteria that has been proposed in 1994 which includes the following: 1- type I hypersensitivity as a reaction to fungal allergens, suggested by skin test or serological tests, 2- nasal polyposis, 3-characteristic findings on CT scan, 4-presence of fungi on direct microscopy or culture, 5 -and allergic mucin containing fungal elements without tissue invasion [28].

Several minor but relevant criteria include a history of asthma, predominance of unilateral disease, radiographic evidence of bone erosion, and peripheral eosinophilia [29]. In 1994 however, the Mayo Clinic experience that was reported by Cody DT, et al. suggested that diagnostic criteria comprise only the presence of allergic mucin and fungal hyphae or a positive fungal culture [30]. That being said, Bent III and Kuhn diagnostic criteria is still considered the most widely accepted criteria for diagnosis of AFRS. To date, there is no definitive management of AFRS. However, a multimodality approach is usually taken, that can be divided into medical and surgical treatment. Both intranasal steroid sprays and oral steroids have been used in the treatment of allergic fungal disease particularly in post-operative period following sinus surgery [14]. Patients often present already on medical therapy for AR and/or CRS when the diagnosis of AFRS is finally made, with no improvement. In AFRS, early surgical intervention is essential to eradicate as much fungi and mucin and prevent future deformations of the facial skeleton as well. Also, subsequent and necessary medical interventions could become more effective [3].

In this article, we will review the literature regarding AFRS cases with similar clinical presentation as our cases to asses for the different presentation of the disease. This includes intraorbital and intracranial extension, association with granuloma, and finally AFRS in the pediatric age group.

\section{AFRS with Intraorbital Extension}

Skull base as well as orbital erosion has been well documented in the literature with co-existence of both in a many cases [31]. The expanding mass leads to bony erosion and involvement of adjacent structures in 6-56\% of AFRS patients [32]. Bozeman, et al. reviewed of 34 patients with complications of AFS, this showed that $38 \%$ of cases had complications related to orbital wall involvement making it the most common. Erosion of the sinus wall was the second most common complication, occurring in $24 \%$ [33].

Ophthalmic complications of sinusitis includes orbital medial wall erosion, proptosis and visual loss in extreme cases [34-37]. The proximity of neurovascular structures to the nasal cavity and sinuses put them at risk of injury, either from the disease process itself or from surgical management. These structures include the internal carotid artery, cranial nerves, cavernous sinus, dura mater and cerebral lobes [38]. The expansion of the disease usually is determined by location of the disease, as well as the path of least resistance. The lamina papyracea is the most common location for bony destruction due to its innate weakness, and the orbit is the most common location for extra sinus disease spread [39].

Some of the proposed mechanisms of visual loss in patients with sinonasal disease include optic neuritis due to an adjacent inflammatory process, central retinal artery occlusion or optic nerve venous congestion due to thrombophlebitis [40]. Several other studies that includes Thakar, et al. [41] and Gupta, et al. [37] along with a case report by Aakalu, et al. [42] have observed good recovery of vision in cases of sudden or recent onset visual loss after surgical decompression and steroid use.

Ashish V, et al. has reported sudden visual loss in one of four patients. Patient had complete visual recovery $(6 / 6)$ due to intravenous steroid administration even before surgical decompression [38]. In our case, patient's visual loss improved post operatively along with use of oral steroids as well.

\section{AFRS with Intracranial Extension}

It is important to note that a chronic, non- tissue invasive, inflammatory process of the nose and paranasal sinuses such as allergic fungal rhinosinusitis (AFRS), will expand with time causing pressure necrosis of bone. This could mimic malignant skull base tumors. Any boneeroding mass in the nasal cavity and/or paranasal sinuses should raise the suspicion of an underlying malignancy, 


\section{Otolaryngology Open Access Journal}

hence should be excluded [39]. In advanced cases of AFRS, lesions may erode the skull base, leading to intracranial presence of mucin as has been described in the literature [39]. Complications in these cases may range from headaches, frontal lobe compression, facial deformity, up to intracranial abscess $[43,44]$. Once AFRS is assumed from the clinical presentation, examination and the Imaging findings, Patient should undergo endoscopic sinus surgery for decompressing the nose and paranasal sinuses along with definitive intraoperative diagnosis, for which biopsy will confirm the non-infiltrative nature of the disease and to rule out malignancies.

Ikram, et al. reported a retrospective study of 26 patients, all whom had AFRS with intracranial spread. 24 underwent minimally invasive endoscopic sinus surgery, only 2 patients however required additional external procedures as pathology was inaccessible endoscopically. None required craniotomy. Postoperatively, all patients received oral steroid therapy for 1 month $(0.5 \mathrm{mg} / \mathrm{kg})$, and remained disease free for a period of up to 5 years [45].

It should also be noted that some rare, extensive cases might require intracranial approach for clearing the intracranial extension, also for possible defect repair that cannot be managed with endoscopic approach alone.
Hence, neurosurgery team in these cases should be consulted for possible intraoperative interference. In our case, patient presented complaining of nasal discharge and obstruction only with no headache, nor any other neurological signs both ophthalmology and neurosurgery team were consulted. Intraoperatively, neurosurgery team were available in case needed small bone erosion was found in the suprasellar, clivusparasellar region which was repaired with haddad flap successfully. Therefore, there was no need for neurosurgery team intervention.

\section{AFRS with Granuloma}

There are several reports present in the literature mentioning different types of fungal sinusitis affecting the same patient. One of the first researches to describe this entity were Zieske, et al. in which they described the occurrence of both invasive and AFS in four of six patients with a clinical diagnosis of AFS [9]. Schubert \& Goetz described one patient with both AFS and fungus ball and another patient with AFS and chronic granulomatous fungal sinusitis [46]. Several reports including Klapper [47], Thakar, et al. [41] and Rupa, et al. [8], Das, et al. [48] also found cases of granuloma coexisting with acute invasive fungal sinusitis (AIFS) and fungal ball (Table 2).

\begin{tabular}{|c|c|c|c|c|}
\hline Author & year & $1^{\text {st }}$ fungal disease & $2^{\text {nd }}$ fungal disease & Total no of cases \\
\hline Zieske, et al. [9] & 1991 & 4 AFRS & CIFS & 4 \\
\hline \multirow{2}{*}{ Klapper, et al. [47] } & \multirow{2}{*}{1997} & $1 \mathrm{AFRS}$ & CGFS & \multirow{2}{*}{2} \\
\hline & & 1 AFRS & CIFS & \\
\hline \multirow{2}{*}{ Schubert \& Goetz [46] } & \multirow{2}{*}{1998} & 1 AFRS & Fungal ball & 1 \\
\hline & & $1 \mathrm{AFRS}$ & CGFS & 1 \\
\hline Thakar, et al. [41] & 2003 & 6 AFRS & CGFS & 6 \\
\hline \multirow{3}{*}{ Das, et al. [48] } & \multirow{3}{*}{2009} & 8 AFRS & CGFS & \multirow{3}{*}{12} \\
\hline & & 1 Fungal ball & CGFS & \\
\hline & & 3 AIFS & CGFS & \\
\hline Rupa, et al. [8] & 2012 & 6 AFRS & CGFS & 6 \\
\hline Chaaban, et al. [50] & 2012 & 1 AFRS & AIFS $^{* *}$ & 1 \\
\hline Gupta, et al. [49] & 2015 & 9 AFRS & CGFS & 57 \\
\hline
\end{tabular}

Table 2: Different cases presented in the literature of AFRS with other types of fungal diseases.

** in this case the patient developed AIFS within 2 weeks of endoscopic sinus surgery for AFRS.

Gupta, et al. in 2015 was the latest which described a series of cases in a retrospective study. 9 out of 57 cases of previously operated AFRS showed presence of granuloma in histology. These were included in the AFS with granuloma Group (group 1) and the rest 48 were included in the AFS group (group 2), they concluded that
Granulomas suggests a more severe disease having higher rate of orbital and skull base erosions [49].

The importance of such studies is to alert the clinician regarding the nature of progression of these diseases, and that the possibility of having "mixed" type of fungal disease does in fact exists. Some even described 


\section{Otolaryngology Open Access Journal}

development of other types of aggressive fungal disease as AIFS post management of AFRS, [50] which should be placed into consideration. Also, in such cases the clinician should expect to have a more extensive bony erosion along with both intracranial and intraorbital extension of disease.

Therefore, Surgeons should be alerted that in the presence of any clinical involvement of the orbit, or any radiological evidence of extrasinus extension could possibly imply the presence of an invasive disease occurring concurrently [41]. In our study, we described one case of a 40 years old male presented complaining of nasal obstruction for 7 years. Examination showed nasal polyps for which he underwent endoscopic sinus surgery. Biopsy was taken intraoperatively that showed inflammatory nasal polyps with multifocal granuloma.

Findings in our case; along with previously mentioned studies would raise suspicion regarding the "noninvasive" nature of AFRS, whether or not the previous proposal that fungal sinusitis should be considered a potentially progressive continuum, where in noninvasive disease may convert to or coexist with an invasive form [26,51].

\section{AFRS in Children}

Nasal polyposis is considered a rare presentation in children unless associated with an underlying pathology like cystic fibrosis or other ciliary dysmotility syndromes $[52,53]$. That being said, AFRS should be considered as a differential as well in a pediatric patient presenting with nasal polyposis, after ruling out the previously mentioned other causes. There are several studies in the literature regarding AFRS in adults. However even though AFRS presents as well but to lesser extent in the pediatric age group, there only a few data in literature regarding its nature, clinical course and recurrence in children [54].

Even though the ratio is relatively equal when accounting for the different age distributions of disease between the genders. When comparing children and adults, there may be an age-related difference in the M/F ratio. A review of children with AFS at University of Texas Southwestern revealed a distinct male predominance, with a 2.1:1 M/F ratio and an average age at diagnosis of 13 years. On the other hand, the adult population showed a female predominance, with an M/F ratio of 1:1.4 [55].

A M/F ratio of 1.5:1 with a mean age at diagnosis of 13.6 years was also reported in an another series of children with AFRS. In a review of 178 pediatric and adult patients whom were diagnosed with AFRS, the following was found:

a) the average time of diagnosis from the initial visit was 11 months

b) patients undergoing an average of 2.4 surgeries before diagnosis.

c) computed tomography was the earliest documented clinical feature most frequently (5.15 months), whereas the latest criterion was a positive fungal smear (8.63 months).

Even though the characteristic computed tomography findings were often the earliest documented signs of disease in patients with AFRS, $65 \%$ of patients didn't have these findings (Melroy and colleagues, unpublished data, 2009) [55]. As for the presentation of AFS is children, it may be quite subtle with the onset typically being an indolent process. Children report a slow onset of nasal airway obstruction and production of large, dark-colored nasal debris $[20,56]$.

However, Patro, et al. presented a prospective study of 50 patients divided into pediatric group (6-14yrs) and adult group (15-65yrs) to compare between the aggressiveness of the disease. It showed that pediatric group had a less mean duration of symptoms, telecanthus and proptosis was more common, and lundmackay radiological scoring was higher. Based on that, they concluded the more aggressive course in pediatric age group when compared to adults with involvement of multiple sinuses [54]. In another prospective study of 200 cases divided into 2 groups. 68 cases in group 1 (less than 15 years) and 132 cases in group 2 (more than 15 years). The study concluded the following: nasal obstruction was the most common presentation in both groups. The children had higher incidence of having unilateral disease compared with adults, and finally group 1 had higher incidence facial deformities, proptosis, intraorbital/intracranial extension along with higher rate of recurrence. Which also suggested a more aggressive nature of AFRS in children when compared to adults as the previous study [56].

Several other studies in the literature has reported alteration in the facial skeleton in $42 \%$ of pediatric patients which includes Proptosis, telecanthus, or malar flattening, compared to $10 \%$ for adults. This could be explained by the more pliable bony structure of children $[20,57,55]$. Also, a case of AFRS in sphenoid sinus of an immunocompetent pediatric patient can present with visual loss. In this case the visual loss was irreversible even after aggressive endoscopic debridement of sinuses and use of corticosteroids. This underscores the 


\section{Otolaryngology Open Access Journal}

potentially serious nature of sphenoid sinusitis and highlights its possibility to induce visual loss [58]. In our Pediatric cases, 6thcase presented with telecanthus and proptosis without any visual impairment during her 1st visit. She improved after undergoing proper management consist of surgical debridement and postoperative both intranasal and oral steroid use.

Whereas the 7thcase was mainly complaining of long standing nasal discharge and on-off headache with no orbital complaint. He also improved after endoscopic sinus surgery with debridement, followed by medical therapy. This shows different presentation of the fungal sinusitis and the need of aggressive intervention for AFRS both medically and surgically for pediatric patients as well.

\section{Conclusion}

AFRS can present clinically in different ways. Despite being categorized as a benign, non-invasive disease, its presentation can range from simple nasal obstruction to signs and symptoms of intraorbital and/or intracranial complications; with paediatric cases being more aggressive in nature than in adults. Other forms of fungal sinusitis may coexist with allergic fungal sinusitis as well. Due to this wide range of differences in clinical presentation, each patient should undergo careful clinical evaluation, detailed histopathological examination to rule out the mixed types, and a lifelong follow up to manage the recurrence.

Data Availability: The data used to support the findings of this study are included within the article. Also, they are available from the corresponding author upon request.

Conflicts of Interest: The authors declare that there is no conflict of interest regarding the publication of this paper.

\section{References}

1. deShazo RD, Chapin K, Swain RE (1997) Fungal sinusitis. N Engl J Med 337(4): 254-259.

2. deShazo RD, O’Brien M, Chapin K, Soto-Aguilar M, Gardner L, et al. (1997) A new classification and diagnostic criteria for invasive fungal sinusitis. Arch Otolaryngol Head Neck Surg 123(11): 1181-1188.

3. Hoyt AEW, Borish L, Gurrola J, Payne S (2016) Allergic Fungal Rhinosinusitis. J Allergy Clin Immunol Pract 4(4): 599-604.
4. Schubert MS (2000) Medical treatment of allergic fungal sinusitis. Ann Allergy Asthma Immunol Off Publ Am Coll Allergy Asthma Immunol 85(2): 90-98.

5. Safirstein BH (1976) Allergic bronchopulmonary aspergillosis with obstruction of the upper respiratory tract. Chest 70(6): 788-790.

6. Marple BF (2009) Allergic Fungal Rhinosinusitis: Current Theories and Management Strategies. The Laryngoscope 111(6): 1006-1019.

7. Katzenstein ALA, Sale SR, Greenberger PA (1983) Allergic Aspergillus sinusitis: a newly recognized form of sinusitis. J Allergy Clin Immunol 72(1): 89-93.

8. Rupa V, Thomas M (2013) Different types of fungal sinusitis occurring concurrently: implications for therapy. Eur Arch Oto-Rhino-Laryngol Off J Eur Fed Oto-Rhino-Laryngol Soc EUFOS Affil Ger Soc OtoRhino-Laryngol - Head Neck Surg 270(2): 603-608.

9. Zieske LA, Kopke RD, Hamill R (1991) Dematiaceous fungal sinusitis. Otolaryngol Head Neck Surg Off J Am Acad Otolaryngol-Head Neck Surg 105(4): 567-577.

10. Sridhara SR, Paragache G, Panda NK, Chakrabarti A (2005) Mucormycosis in immunocompetent individuals: an increasing trend. J Otolaryngol 34(6): 402-406.

11. Suryanarayan Rao S, Panda NK, Pragache G, Chakrabarti A, Saravanan K (2006) Sinoorbital mucormycosis due to Apophysomyces elegans in immunocompetent individuals--an increasing trend. Am J Otolaryngol 27(5): 366-369.

12. Taxy JB, El-Zayaty S, Langerman A (2009) Acute fungal sinusitis: natural history and the role of frozen section. Am J Clin Pathol 132(1): 86-93.

13. Hamilos DL (2000) Chronic sinusitis. J Allergy Clin Immunol 106(2): 213-227.

14. Chang C, Gershwin ME, Thompson GR (2013) Fungal disease of the nose and sinuses: an updated overview. Curr Allergy Asthma Rep 13(2): 152-161.

15. Morpeth JF, Rupp NT, Dolen WK, Bent JP, Kuhn FA (1996) Fungal sinusitis: an update. Ann Allergy Asthma Immunol 76(2): 128-139.

16. Schubert MS (2006) Allergic fungal sinusitis. Clin Rev Allergy Immunol 30(3): 205-216. 


\section{Otolaryngology Open Access Journal}

17. Manning SC, Holman M (1998) Further evidence for allergic pathophysiology in allergic fungal sinusitis. Laryngoscope 108(10): 1485-1496.

18. Kim C, Kacker A, Chee R-I, Lelli GJ (2013) Allergic Fungal Sinusitis Causing Nasolacrimal Duct Obstruction. Orbit 32(2): 143-145.

19. Katzenstein AL, Sale SR, Greenberger PA (1983) Pathologic findings in allergic aspergillus sinusitis. A newly recognized form of sinusitis. Am J Surg Pathol 7(5): 439-443.

20. McClay JE, Marple B, Kapadia L, Biavati MJ, Nussenbaum B, et al. (2002) Clinical presentation of allergic fungal sinusitis in children. Laryngoscope 112(3): 565-569.

21. Mukherji SK, Figueroa RE, Ginsberg LE, Zeifer BA, Marple BF, et al. (1998) Allergic fungal sinusitis: CT findings. Radiology 207(2): 417-422.

22. Dutre T, Al Dousary S, Zhang N, Bachert C (2013) Allergic fungal rhinosinusitis (AFRS) - more than a fungal disease? Clin Transl Allergy 3(2): 15.

23. Kupferberg SB, Bent JP, Kuhn FA (1997) Prognosis for allergic fungal sinusitis. Otolaryngol Head Neck Surg 117(1): 35-41.

24. Harner SG, Corey JP, Delsupehe KG, Ferguson BJ (1995) Allergic Fungal Sinusitis: Allergic, Infectious, or Both? Otolaryngol Neck Surg 113(1): 110-119.

25. Kuhn FA, Javer AR (2000) Allergic fungal rhinosinusitis: Perioperative management, prevention of recurrence, and role of steroids and antifungal agents. Otolaryngol Clin North Am 33(2): 419-433.

26. Hartwick RW, Batsakis JG (1991) Sinus aspergillosis and allergic fungal sinusitis. Ann Otol Rhinol Laryngol 100(5): 427-430.

27. Manning SC, Merkel M, Kriesel K, Vuitch F, Marple B (1997) Computed tomography and magnetic resonance diagnosis of allergic fungal sinusitis. Laryngoscope 107(2): 170-176.

28. Bent JP, Kuhn FA (1994) Diagnosis of allergic fungal sinusitis. Otolaryngol--Head Neck Surg Off J Am Acad Otolaryngol-Head Neck Surg 111(5): 580-588.
29. Willard CC, Eusterman VD, Massengil PL (2003) Allergic fungal sinusitis: report of 3 cases and review of the literature. Oral Surg Oral Med Oral Pathol Oral Radiol Endod 96(5): 550-560.

30. Cody DT, Neel HB, Ferreiro JA, Roberts GD (1994) Allergic fungal sinusitis: the Mayo Clinic experience. Laryngoscope 104(9): 1074-1079.

31. Marfani MS, Jawaid MA, Shaikh SM, Thaheem K (2010) Allergic fungal rhinosinusitis with skull base and orbital erosion. J Laryngol Otol 124(2): 161-165.

32. Ghegan MD, Lee F-S, Schlosser RJ (2006) Incidence of skull base and orbital erosion in allergic fungal rhinosinusitis (AFRS) and non-AFRS. Otolaryngol Head Neck Surg Off J Am Acad Otolaryngol Head Neck Surg 134(4): 592-595.

33. Bozeman S, deShazo R, Stringer S, Wright L (2011) Complications of allergic fungal sinusitis. Am J Med 124(4): 359-368.

34. Dunlop IS, Billson FA (1988) Visual failure in allergic aspergillus sinusitis: case report. $\mathrm{Br} \mathrm{J}$ Ophthalmol 72(2): 127-130.

35. Carter KD, Graham SM, Carpenter KM (1999) Ophthalmic manifestations of allergic fungal sinusitis. Am J Ophthalmol 127(2): 189-195.

36. Marple BF, Gibbs SR, Newcomer MT, Mabry RL (1999) Allergic fungal sinusitis-induced visual loss. Am J Rhinol 13(3): 191-195.

37. Gupta AK, Bansal S, Gupta A, Mathur N (2007) Visual loss in the setting of allergic fungal sinusitis: pathophysiology and outcome. J Laryngol Otol 121(11): 1055-1059.

38. Vashishth A (2015) Extensive Allergic Fungal Rhinosinusitis: Ophthalmic and Skull Base Complications. Indian J Otolaryngol Head Neck Surg 67(3): 227-233.

39. Meccariello G, Deganello A, Mannelli G, Bianco G, Ammannati F, et al. (2013) Allergic fungal rhinosinusitis infiltrating anterior skull base and clivus. Auris Nasus Larynx 40(4): 405-408.

40. Patt BS, Manning SC (1991) Blindness resulting from orbital complications of sinusitis. Otolaryngol Head Neck Surg 104(6): 789-795. 


\section{Otolaryngology Open Access Journal}

41. Thakar A, Lal P, Dhiwakar M, Bahadur S (2011) Optic nerve compression in allergic fungal sinusitis. J Laryngol Otol 125(4): 381-385.

42. Aakalu VK, Sepahdari A, Kapur R, Setabutr $P$, Putterman AM, et al. (2009) Allergic Fungal Sinusitis Induced Visual Loss and Optic Neuropathy. NeuroOphthalmol 33(6): 327-332.

43. Holbrook JF, Eastwood JD, Kilani RK (2014) Intracranial abscess as a complication of allergic fungal sinusitis. J Neuroimaging Off J Am Soc Neuroimaging 24(1): 95-98.

44. Nussenbaum B, Marple BF, Schwade ND (2001) Characteristics of bony erosion in allergic fungal rhinosinusitis. Otolaryngol Head Neck Surg 124(2): 150-154.

45. Ikram M, Akhtar S, Ghaffar S, Enam SA (2008) Management of allergic fungal sinusitis with intracranial spread. Eur Arch Oto Rhino Laryngol 265(2): 179-184.

46. Schubert MS, Goetz DW (1998) Evaluation and treatment of allergic fungal sinusitis. I. Demographics and diagnosis. J Allergy Clin Immunol 102(3): 387394.

47. Klapper SR, Lee AG, Patrinely JR, Stewart M, Alford EL (1997) Orbital involvement in allergic fungal sinusitis. Ophthalmology 104(12): 2094-2100.

48. Das A, Bal A, Chakrabarti A, Panda N, Joshi K (2009) Spectrum of fungal rhinosinusitis; histopathologist's perspective. Histopathology 54(7): 854-859.

49. Gupta R, Gupta AK, Patro SK, Yadav J, Chakrabarti A, et al. (2015) Allergic fungal rhino sinusitis with granulomas: A new entity? Med Mycol 53(6): 569575.
50. Chaaban MR, Bell W, Woodworth BA (2013) Invasive mucormycosis in an immunocompetent patient with allergic fungal rhinosinusitis. Otolaryngol Head Neck Surg 148(1): 174-175.

51. Rowe-Jones J (1993) Paranasal aspergillosis-a spectrum of disease. J Laryngol Amp Otol 107(9): 773-774.

52. Yung MW, Gould J, Upton GJG (2002) Nasal polyposis in children with cystic fibrosis: a long-term follow-up study. Ann Otol Rhinol Laryngol 111(12): 1081-1086.

53. Do BAJ, Lands LC, Saint-Martin C, Mascarella MA, Manoukian JJ, et al. (2014) Effect of the F508del genotype on outcomes of endoscopic sinus surgery in children with cystic fibrosis. Int J Pediatr Otorhinolaryngol 78(7): 1133-1137.

54. Patro SK, Verma RK, Panda NK, Chakrabarti A (2015) Understanding paediatric allergic fungal sinusitis: Is it more aggressive? Int J Pediatr Otorhinolaryngol 79(11): 1876-1880.

55. Thorp BD, McKinney KA, Rose AS, Ebert CS (2012) Allergic fungal sinusitis in children. Otolaryngol Clin North Am 45(3): 631-642.

56. Gupta AK, Bansal S, Gupta A, Mathur N (2006) Is fungal infestation of paranasal sinuses more aggressive in pediatric population? Int J Pediatr Otorhinolaryngol 70(4): 603-608.

57. Campbell JM, Graham M, Gray HC, Bower C, Blaiss MS, et al. (2006) Allergic fungal sinusitis in children. Ann Allergy Asthma Immunol 96(2): 286-290.

58. Herrmann BW, White FV, Forsen JW (2006) Visual loss in a child due to allergic fungal sinusitis of the sphenoid. Otolaryngol Head Neck Surg 135(2): 328329. 